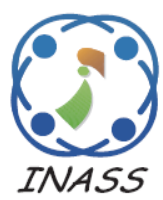

\title{
Image Restoration in Noisy Free Images Using Fuzzy Based Median Filtering and Adaptive Particle Swarm Optimization - Richardson-Lucy Algorithm
}

\author{
Narendra Kumar ${ }^{1 *}$ \\ Hari Shanker Shukla ${ }^{2}$ \\ ${ }^{1,2}$ Deen Dayal Upadhyaya Gorakhpur University, Gorakhpur, India \\ ${ }^{3}$ Graphic Era university, Dehradun, Uttarakhand, India \\ *Corresponding author's Email: narendrakumar1082@gmail.com
}

Rakesh Prakash Tripathi ${ }^{3}$

\begin{abstract}
In this paper, we have proposed adaptive methods for image restoration in which the input images are affected by noise which is removed by fuzzy based median filter (FMF). The noise removed images from the FMF is appears to be so there is a need to restore the images with high quality. To restore the images an APSO (Adaptive particle swarm optimization) based Richardson-Lucy (R-L) algorithm is utilized. By both FMF and APSO-RL methods the denoising and restoration of the image is performed efficiently. The performance of the image denoising and restoration technique is evaluated by comparing the result of proposed technique with the existing denoising filter and GA, PSO methods. The comparison result shows a high-quality denoising and restoration ratio for the noisy images than the existing methods, in terms of peak signal-to-noise ratio (PSNR) and second-derivative-like measure of enhancement (SDME).
\end{abstract}

Keywords: Image denoising; Image restoration; Particle Swarm Optimization; Median filter; Adaptive Particle Swarm Optimization (APSO); Richardson-Lucy (R-L) algorithm.

\section{Introduction}

In this modern digital age, many applications are based on images and therefore the resulting achievements must rely on their quality. Since images are not always in a good quality due to various types of noise (natural noise, defects in the sensors, transmission problems, etc.), it is important to eliminate the noise automatically and efficiently [1]. Digital image processing techniques now are used to solve a variety of problems. Although often unrelated, these problems commonly require methods capable of enhancing pictorial information for human interpretation and analysis [2]. Though, the technologies used to improve resolution and quality of noisy images remains an issue in many medical images applications. Removing noise in these digital images remains as one of the major challenges in the study of medical imaging [3]. In principle, the restoration task can be understood as an inverse problem, i.e. one can attack it by solving a related variational problem [4]. The purpose of image restoration is to estimate the original image from the degraded data. It is widely used in various fields of applications, such as medical imaging, astronomical imaging, remote sensing, microscopy imaging, photography deblurring, and forensic science, etc. Restoration is beneficial to interpreting and analyzing the remote sensing images. After restoration, the blur phenomenon of the images is reduced $[5,6]$. Restoration is known to be an ill-posed inverse problem, since the deconvolution requires the inverse of the blurring operator, which may be nearly singular or even not exist, resulting in magnification of noise [7].The act of restoring an image to remove noise and blur is typically an under-constrained problem. Information lost during a lossy observation process needs to be restored with prior information about natural images to achieve visual realism [8]. Image blur and noise are the most common problems in photography, which can be especially significant in light limited situations, resulting in a ruined photograph [9]. Consequently the restoration techniques are oriented towards modeling the 
degradation and applying the inverse process in order to recover the original image $[10,11]$. The objective of denoising is to remove the noise effectively while preserving the original image details as much as possible. So far, many approaches have been proposed to get rid of noise [12]. Traditionally, this is achieved by linear processing such as Wiener filtering $[13,14-15]$. The image denoising is important, not only because of the evident applications it serves. Being the simplest possible inverse problem, it provides a convenient platform over which image processing ideas and techniques can be assessed [16].

The development of variational partial differential Equation based on image restoration techniques offer a new thought to address the problem about image denoising and image edge preserve. It has become the research hotspot in recent years $[17,18]$. Many conventional image processing algorithms are based on the assumption of local structural regularity, which states that there are meaningful structures in the spatial space of natural images [19]. Using redundant representations and sparsity as driving forces for denoising of signals has drawn a lot of research attention in the past decade or so [20].

The reminder of this paper is organized as follows. In section 2, a brief discussion about the research works related to the image denosing and restoration is given. In section 3 the problem statement and the contribution of our proposed method has been described. Our proposed noise removal and restoration is briefly explained in section 4 . The implementation results and conclusion of the paper is given in section 5 and 6 .

Fuzzy is one of the efficient technique evolved in several research purposes, so in our proposed method we are employing efficient cascade techniques to filter out noise and for restoration of images in data base. Noisy pixels are marked and removed at the filter process and in the later stage pixels are enhanced to standard level.

The advantage of the proposed method can be clearly noticed from the throughput of proposed and existing techniques.

\section{Related Works}

Michael et al. [21] have developed model for restoration of blurred images from the pixels those were missing in Cartoon-Plus Textured images. The image used in their work was a combination of cartoon and texture and hence they have used total variation norm and its dual norm for regularizing the cartoon and the texture separately. In order to decompose the cartoon and texture from blurred images, they have used an efficient numerical algorithm with guaranteed convergence.

Weisheng et al. [22] have proposed and developed a sparse representation model for restoration of images. Usually sparse representations have considered images as the collections of few atoms that has been chosen from an over-complete dictionary. But those traditional sparse representations were not effective to reconstruct degraded images into original images. To enhance the performance of image restoration using sparse representation, in this work they have used sparse coding noise and image restoration has almost concentrated on reducing this sparse code noise in degraded images using nonlocally centralized sparse representation.

Ajith et al. [23] have proposed a image denoising technique using Higher Order Singular Value Decomposition (HOSVD). Their denoising technique is considered to be very simple and was patch-based machine learning technique. In their work they have grouped the similar noisy patches from the noisy images into a single 3-dimensional stack and then have computed the HOSVD coefficients for those noisy patch stacks. These HOSVD coefficients were manipulated by thresholding and these thresholded HOSVD coefficients were inverted to produce final original image.

Abdolhossein et al. [24] have proposed an optimum adaptive wavelet packet (WP) technique for denoising images. Their adaptive technique of thresholding was based on Gaussian distribution. In order to obtain optimal wavelet bases from noisy images, the authors of this work have utilized a computationally efficient multilevel wavelet basis using Shannon entropy. This has selected an adaptive thresholding value which was the statistical value of its subband coefficients.

T. S. Cho et al. [25] have proposed an image restoration method based on iterative distribution reweighting (IDR) which is a deconvolution method. This has imposed a global constraint on the gradient of the images, which led to a gradient distribution of restored images similar to that of the gradient distribution of reference images. The image restoration technique they have used is an alternative image restoration technique for reconstructing images. In this they have penalized gradients based on a fixed gradient prior and have compared the reconstructed image's gradient distribution with desired gradient distribution and have selected images that have gradient distribution similar to desired gradient distribution.

P. Zhang and F. Li [26] have proposed a new 
adaptive weighted mean filter (AWMF) for detecting and removing high level of salt-and-pepper noise. For each pixel, they firstly determined the adaptive window size by continuously enlarging the window size until the maximum and minimum values of two successive windows were equal respectively. Then the current pixel was regarded as noise candidate if it was equal to the maximum or minimum values, otherwise, it was regarded as noise-free pixel.

V. B. S. Prasath et al. [27] have proposed a spatially adaptive multiscale variable exponent-based anisotropic variational PDE method that overcomes current shortcomings, such as over smoothing and stair casing artifacts, while still retaining and enhancing edge structures across scale. Their innovative model automatically balances between Tikhonov and Total Variation (TV) regularization effects using scene content information by incorporating a spatially varying edge coherence exponent map constructed using the eigen values of the filtered structure tensor.

H. Zhang et al. [28] introduced a new HSI restoration method based on low-rank matrix recovery (LRMR), which can simultaneously remove the Gaussian noise, impulse noise, deadlines, and stripes. By lexicographically ordering a patch of the HSI into a 2-D matrix, the low-rank property of the hyperspectral imagery was explored, which suggested that a clean HSI patch can be regarded as a low-rank matrix.

A. Roy et al. [29], support vector machine (SVM) classification based Fuzzy filter (FF) was proposed for removal of impulse noise from gray scale images. When an image was affected by impulse noise, the quality of the image was distorted since the homogeneity among the pixels was broken. SVM was incorporated for detection of impulse noise from images.

R. Dash and B. Majhi [30], have proposed a deal with estimation of parameters for motion blurred images. The objectives were to estimate the length (L) and the blur angle $(\theta)$ of the given degraded image as accurately as possible so that the restoration performance can be optimised. Gabor filter is utilized to estimate the blur angle whereas a trained radial basis function neural network (RBFNN) estimates the blur length.

\section{The Problem Statement}

Section 2, reviews the recent research works related to the image denosing and restoration techniques. In those methods, wavelets have provided better response for image and hence researchers have introduced many wavelet based algorithms for image denoising. The performance of wavelets was due to their sparsity and multiresolution structure. The basic denoising algorithms namely spatial denoising methods and transform denoising methods have proved enough in field of denoising. Yet they are lacking performance in some areas which could be improved. The disadvantage of spatial filters is that these filters not only smooth the data to reduce noise but also blur edges in image.

Linear filters are also not effective against signal dependent noises for image denoising. Similarly spatial frequency filtering and wavelet transform based algorithms for image denoising are lot more time consuming and are computationally complex to be implemented. If all the aforesaid drawbacks in the literary works are solved, then the image denoising and quality is improved with higher efficiency.

Inspite of smoothing the data for the filtering purpose, the proposed method mark the noisy and noise free pixels from the image. Afterward by using the filtering window the noise free image is generated.

\section{Contributions of the paper}

The main contributions of the paper are,

- Remove the noise from the images by using Fuzzy based Median Filter (FMF).

- Restore the noise removed images by APSO-RL algorithm.

\section{Proposed Image Denoising and Restoration Technique}

Here, an image denoising (FMF) and restoration (APSO-RL) techniques are proposed for the images which have affected by noises. Our proposed technique mainly comprised of two processing stages namely,

- Noise removal by FMF

- Image Restoration using APSO-RL

These two stages are consecutively applied on the noisy images and obtain the noise free and restored images. The proposed technique structure is illustrated in Fig. 1. 


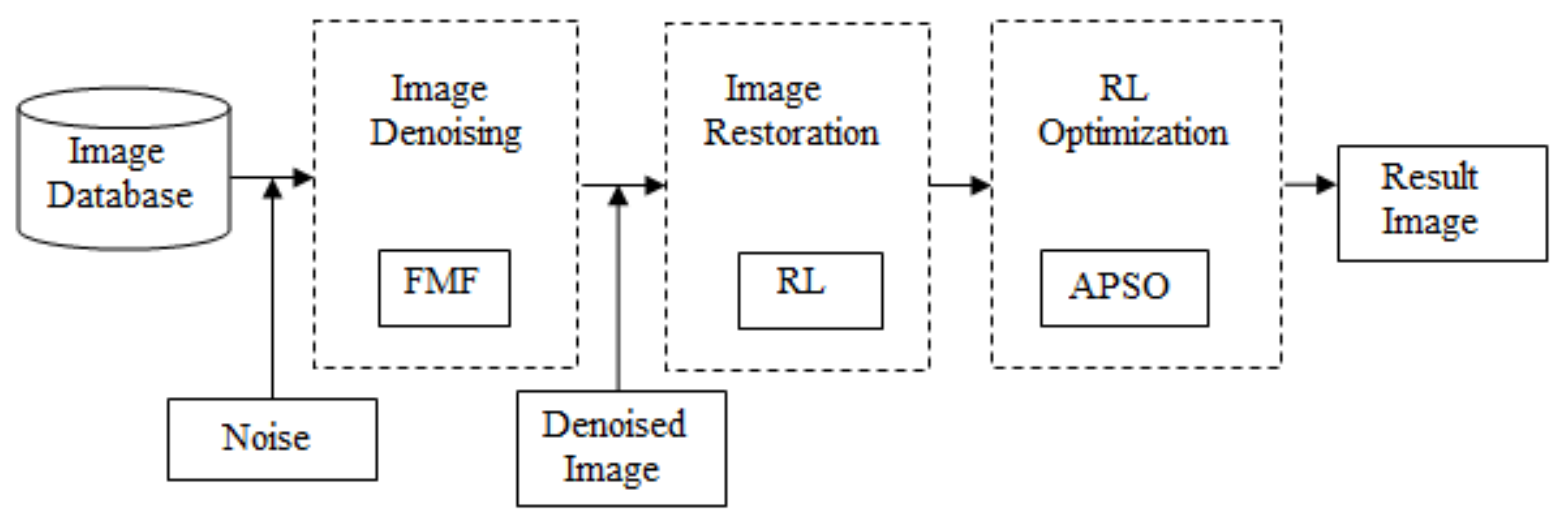

Figure.1 Structure of our proposed image denoising and restoration by using FMF and APSO-RL

Let we considered an image database $I D=\{O(i, j), A(i, j)\}$, where, $O \leq i \leq M-1,0 \leq j \leq \mathrm{N}-1, O(i, j)$ and $A(i, j)$ be the original and noise affected images respectively. At first, the given noise image $A(i, j)$ is given to the image denoising process and that noise removed images are send to the image restoration process. The process of image denoising and restoration are briefly explained in the following sections 4.1 and 4.2 .

\subsection{Image Denoising using FMF}

In image denoising the given input noisy image $A(i, j)$ is to be processed by exploiting the fuzzy based median filter. Initially in FMF a mask is created to mark the noisy and noise free pixels from the image. Afterward by using the filtering window and fuzzy membership functions the noise free image is generated. The process of image denoising by fuzzy based median filter (FMF) is given below.

Step 1: Initially a binary mask $M(i, j)$ is created to point out the noise pixels in the image $A(i, j)$ by using

$$
M(i, j)= \begin{cases}0, & p(i, j)=255 \text { or } 0 \\ 1, & \text { otherwise }\end{cases}
$$

In Eq. (1), $p(i, j)$ is the pixel at location $(i, j)$ with intensity $p$. Where $p(i, j)=1$ denotes the noise free pixel and $p(i, j)=0$ represents the noise pixel from the image is given to the next filtering process.

Step 2: next an adaptive size filtering window $w(i, j)$ is designed with the dimensions $(2 d+1) \times(2 d+1)$, is given as,

$$
\begin{aligned}
& w(i, j)=\{p(i+k, j+l)\} ; \\
& k, l \in\{-d, d\}
\end{aligned}
$$

Where $w$ denotes window and $(i, j)$ represents location, $d$ is a nonzero positive integer. The noise pixel is detected means, $d$ is initialized to one, i.e. $w(i, j)$ initiate the filtering process with a window of size $3 \times 3$.
Step 3: The number of noise free pixels $F(i, j)$ is computed by counting the number of $1 \mathrm{~s}$ in $M(i, j)$ is calculated as,

$$
F(i, j)=\sum_{k, l \in(-d, d)} M(i+k, j+l)
$$

Where $F$ denotes the free pixels in location $(i, j)$. Step 4: If $F(i, j)<1$ means the current window $w(i, j)$ does not have a minimum number of one's (noise free pixels). So we expand the window size by one at each of its four sides.

Step 5: Repeat the steps 3 and 4 until the criterion $F(i, j)>1)$ is satisfied.

Step 6: Find median value by using noise free pixels in the current window $w(i, j)$, which is given as,

$$
\begin{aligned}
& M^{\prime}(i, j)=\text { median }\{p(i+k, j+l)\} ; \\
& M(i+k, j+l)=1
\end{aligned}
$$

Step 7: Afterward, the local information from $w(i, j)$ is extracted by computing the maximum absolute luminance difference is stated as,

$$
\begin{aligned}
& D(i, j)=\max \{d(i+m, j+n)\} \\
& =|p(i+m, j+n)-p(i, j)| \\
& (i+m, j+n) \neq(i, j)
\end{aligned}
$$

Step 8: In FMF the fuzzy reasoning is applied to the extracted local information. The defined fuzzy membership function $F z(i, j)$,

$$
F z(i, j)= \begin{cases}0 & : D(i, j)<T_{1} \\ \frac{D(i, j)-T_{1}}{T_{2}-T_{1}} & : T_{1} \leq D(i, j)<T_{2} \\ 1.0 & : D(i, j) \geq T_{2}\end{cases}
$$

In Eq. (6), $T_{1}$ and $T_{2}$ are two predefined threshold values.

Step 9: Finally we compute denoised image $A^{\prime}(i, j)$ as follows,

$$
A^{\prime}(i, j)=F z(i, j) \times M^{\prime}(i, j)+[1-F z(i, j) \times p(i, j)]
$$

Where, $A(i, j)$ is denoised image, $F z$ is fuzzy function, $M$ is median.

By performing the aforementioned fuzzy based 
filtering algorithm we get an noise free image $A^{\prime}(i, j)$.

\subsection{Image Restoration by APSO-RL}

After performing the denoising process, the noise free image $A^{\prime}(i, j)$ is given to the image restoration to enhance the image quality. To restore the image, Richardson-Lucy algorithm is utilized, in that RL algorithm the restoration performance is improved by optimizing the point spread function (PSF) in the RL algorithm by APSO. The RL algorithm and the APSO are explained in following sub sections.

\subsubsection{Richardson-Lucy algorithm}

The Richardson-Lucy (R-L) algorithm uses a probabilistic approach for recovering the degraded image. Here, when the denoisied image $A^{\prime}(i, j)$ is given as input, an image $\mathrm{k}$ that maximizes the probability of observing the image $A^{\prime}(i, j)$ is found out. Considering the image as an observation of a Poisson process, the likelihood function is defined as:

$$
\begin{aligned}
& p\left(A^{\prime}(i, j) \mid k\right)= \\
& \prod_{z} \frac{h(z) \times k(z) A^{A^{\prime}(i, j)(z)} e^{-h(z) k(z)}}{A^{\prime}(i, j)(z) !}
\end{aligned}
$$

From this Equation, a functional to be minimized,

$$
L(\hat{k})=-\log p\left(A^{\prime}(i, j) \mid k\right)
$$

$L(\hat{k})$ is obtained, giving the maximum likelihood estimation as:

$$
L(\hat{k})=\sum_{z}-A^{\prime}(i, j)(z) \cdot \log [(h \times \hat{k})(z)]+(h \times \hat{k})(z)
$$

An iterative algorithm can be derived from the above functional. It is called the Richardson-Lucy algorithm and it is given by:

$$
\hat{k}_{n+1}(z)=\left[\left(\frac{A^{\prime}(i, j)(z)}{\hat{k}(z) \times h(z)}\right) \times h(z)\right] \times \hat{k}_{n}(z)
$$

This algorithm stops after a finite number of iterations. When the deconvolution is ill-posed, a common situation in real applications, the signal-tonoise ratio becomes increasingly poorer as the number of iterations $n \rightarrow \infty$. In RL algorithm, set of operations is carried out for fixed number of iterations and finally we have the estimated enhanced, restored image $A^{\prime \prime}(i, j)$. The optimized PSF by our proposed APSO is helpful to the RL algorithm for recovering the blurred image.

\subsubsection{PSF optimization by APSO}

The APSO in PSF computation is described
* Initialization: Initially the particles $p_{i} ; 0 \leq i \leq n$ is generated, where $n$ is the total number of generated particles which is generated in the range of $[0,1]$. In our case, 10 matrices are randomly generated to form the initial population. These generated particles are evaluated based on their fitness function which is sated below.

* Parameters: In APSO, the particles position, velocity, learning parameters, inertia, weight and maximum number of iterations are defined.

* Fitness: Every particle's fitness value is calculated by using the formula given, $S D M E=$

$$
-\frac{1}{b_{1} \cdot b_{2}} \sum_{i=1}^{b_{1}} \sum_{j=1}^{b_{2}} 20 \ln \left|\frac{p_{\max , j, i}-2 p_{c e n, j, i}+p_{\min , j, i}}{p_{\max , j, i}+2 p_{c e n, j, i}+p_{\min , j, i}}\right|
$$

SDME is an enhancement measure integrating the idea of the second-derivative. Suppose the image $A^{\prime}(i, j)$ is divided into $b_{1} \times b_{2}$ blocks, and $p_{\max }, j, i$, $p_{\min }, j, i$, are the maximum and minimum values of the pixels in each block separately and $p_{c e n}, j, i$ is the intensity of the center pixel in each block. The particles that have minimum fitness value is selected as the best particles.

* Velocity and Position: Based on the pbest and gbest values, the particles velocity and positions are updated by exploiting the Equations stated below,

$$
\begin{aligned}
& V_{i}^{(n+1)}= \\
& \omega^{n} V_{i}^{n}+c_{1} \cdot r_{1} \cdot\left(p_{i}-x_{i}^{(n)}\right)+c_{2} \cdot r_{2} \cdot\left(g_{i}-x_{i}^{(n)}\right) \\
& \quad x_{i}^{(n+1)}=x_{i}^{(n)}+\delta V_{i}^{(n+1)}
\end{aligned}
$$

In Eq. (13) $c_{1}, c_{2}$ are the learning factors, $\omega$ and $\delta$ represents the inertial weight and constraint factor, rand is positive random number between 0 and $1, V_{i}^{(n)}$ is the velocity of $i^{\text {th }}$ particle at iteration $\mathrm{n}, x_{i}^{(n)}$ is the current position of the particle $i$ at iteration $\mathrm{n}, p_{i}$ is the position of the best fitness value of the particle at the current iteration and $g_{i}$ is the position of the particle with the best fitness value in the swarm. In the existing PSO the acceleration coefficients values are fixed as constant. To improve the PSO performance the constant values are modified by,

$$
\begin{gathered}
m c_{1}=\left(c_{1 f}-c_{1 i}\right) \times \frac{I}{M I}-c_{1 i} \\
m c_{2}=\left(c_{2 f}-c_{2 i}\right) \times \frac{I}{M I}-c_{2 i}
\end{gathered}
$$

Where, $c_{l f}, c_{2 f}, c_{l i}, c_{2 i}$ are final and initial value of $c_{1 i}$ and $c_{2 i}$ respectively. $I$ is the current iteration and $M I$ is the maximum number of iterations. below, 
* New Particles Updation: The new particles values from Eq. (13) and (14) are given to the fitness value computation process.

* Stopping Criteria: The process is repeated until the maximum number of iterations is reached. Thus the optimized PSF from the AGA is utilized in the RL algorithm for recovering the blurred image more effectively.

\section{Experimental Results and Discussion}

The proposed image denoising and restoration technique is implemented in the working platform of MATLAB with machine configuration as follows,

Processor: Pentium(R) Dual-Core

OS: Microsoft Windows Xp

CPU speed: $2.70 \mathrm{GHz}$

RAM: $0.99 \mathrm{~GB}$

In our proposed method, the noisy images from the database are given to the image denoising and restoration process by using the techniques AMF and APSO-RL. The sample images from the database are given Fig. 2

In these database images noises are added and further utilized in the image denoising process. The noise added database images are shown in Fig. 3.

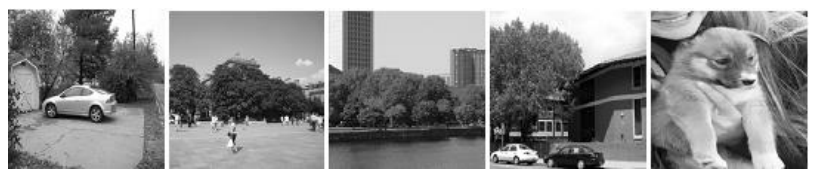

Figure.2 Sample images from the database
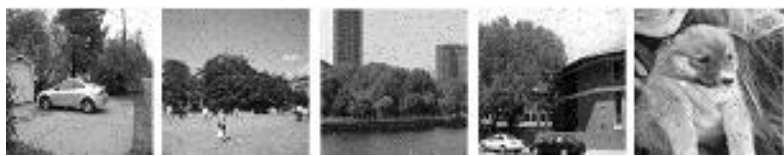

Figure.3 Noise added images
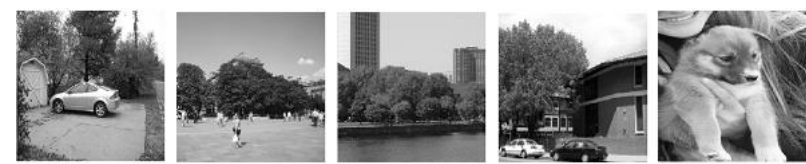

Figure.4 Denoised result images from FMF
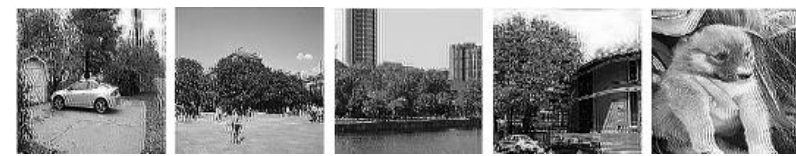

Figure.5 Restored result images from APSO-RL
Those noises added images are given in Fig. 3 are given to the image denoising process. In our proposed method fuzzy based median filter (FMF) is utilized in the noise removal process. Thus the noise removed images from the FMF is given in Fig. 4.

To improve the image quality and restore the pixels, the noisy free images are forward to the image restoration process. Here we have utilized an RL algorithm for the restoration process, during the restoration the RL algorithm is optimized by the APSO optimization method. The restored result images from the APSO-RL are shown in Fig. 5.

Moreover our proposed image denoising and restoration performance is analyzed by the performance metrics like PSNR and SDME.

\subsection{Performance Analysis}

Our proposed method performance is analyzed using performance measures like PSNR and SDME by varying the noise variance as $0.02,0.04,0.06$ and 0.08. The proposed FMF image denoising method performance is evaluated and compared with the existing denoising filters. The performance results and the comparison graph are given in Table 1 and Fig.6.

As can be seen from Fig.6, our proposed FMF based image denoising method has given high PSNR denoised image than the other denoising filtering methods. The noise variance at 0.02 our proposed FMF filter has given high PSNR value than the other methods when increasing the noise variance the PSNR value of the denoised image is decreased but it is not lower than existing filtering methods. Hence, our proposed FMF based image denoising method has given high performance results in removing the noise from the images.

In second stage, the image restoration is performed on the noise free images by using the APSO-RL algorithm. This image restoration performance is analyzed and compared with the other optimization methods GA, PSO and different noise removal filtering methods like bilateral, wiener and median filters respectively. The image restoration performance result in terms of SDME measure of our APSO-RL and PSO-RL is given in Table 2 and the consequent average performance comparison graph is shown in Fig. 7. 
Table 1. Proposed FMF and different filtering methods PSNR value of four Different Impulse Noise Variance levels $0.02,0.04,0.06$ and 0.08

\begin{tabular}{|c|c|c|c|c|c|}
\hline $\begin{array}{c}\text { Noise } \\
\text { Variance } \\
\left(\sigma^{2}\right)\end{array}$ & Images & $\begin{array}{l}\text { Proposed } \\
\text { FMF(in dB) }\end{array}$ & $\begin{array}{c}\text { Bilateral } \\
\text { Filter (in } \\
\text { dB) }\end{array}$ & $\begin{array}{c}\text { Wiener } \\
\text { Filter (in } \\
\text { dB) }\end{array}$ & $\begin{array}{c}\text { Median } \\
\text { Filter (in } \\
\text { dB) }\end{array}$ \\
\hline \multirow{10}{*}{0.02} & 1 & 87.2874 & 70.0543 & 71.6009 & 72.0451 \\
\hline & 2 & 91.3327 & 72.2855 & 71.9249 & 74.7118 \\
\hline & 3 & 84.4181 & 69.8112 & 71.1573 & 71.7993 \\
\hline & 4 & 77.0010 & 67.6669 & 71.7240 & 69.8711 \\
\hline & 5 & 87.5382 & 70.2915 & 71.4029 & 71.4261 \\
\hline & 6 & 90.7962 & 72.5384 & 72.1925 & 74.9932 \\
\hline & 7 & 81.1700 & 69.2943 & 70.8742 & 70.9295 \\
\hline & 8 & 89.5547 & 72.6549 & 71.3827 & 73.1465 \\
\hline & 9 & 87.1535 & 71.7058 & 72.3107 & 72.8806 \\
\hline & 10 & 79.7139 & 69.3385 & 71.3172 & 70.6879 \\
\hline \multirow{10}{*}{0.04} & 1 & 84.4846 & 69.9548 & 69.9415 & 71.9551 \\
\hline & 2 & 89.1186 & 72.1021 & 70.5724 & 74.6529 \\
\hline & 3 & 82.8733 & 69.7037 & 69.7528 & 71.8104 \\
\hline & 4 & 76.4389 & 67.5795 & 70.2744 & 69.6664 \\
\hline & 5 & 84.4065 & 70.2184 & 70.3083 & 71.3504 \\
\hline & 6 & 88.0083 & 72.3879 & 70.7002 & 74.8326 \\
\hline & 7 & 79.9017 & 69.1603 & 69.1538 & 70.7439 \\
\hline & 8 & 87.0027 & 72.4721 & 69.8783 & 73.0636 \\
\hline & 9 & 84.9880 & 71.5845 & 70.7029 & 72.5996 \\
\hline & 10 & 78.8971 & 69.2571 & 69.9906 & 70.4853 \\
\hline \multirow{10}{*}{0.06} & 1 & 82.7463 & 69.8272 & 69.0588 & 71.8151 \\
\hline & 2 & 86.9377 & 71.8754 & 69.7109 & 74.3603 \\
\hline & 3 & 81.4041 & 69.5771 & 69.0264 & 71.4869 \\
\hline & 4 & 75.8496 & 67.4946 & 69.4270 & 69.4714 \\
\hline & 5 & 83.3707 & 70.1314 & 69.6541 & 71.2568 \\
\hline & 6 & 86.4710 & 72.2625 & 70.0404 & 74.6951 \\
\hline & 7 & 79.2214 & 69.0378 & 68.4798 & 70.5557 \\
\hline & 8 & 85.4588 & 72.2125 & 69.0254 & 72.9287 \\
\hline & 9 & 83.7731 & 71.4179 & 69.8563 & 72.4062 \\
\hline & 10 & 78.2927 & 69.1259 & 69.1517 & 70.4512 \\
\hline \multirow{10}{*}{0.08} & 1 & 81.1445 & 69.6454 & 68.4680 & 71.7363 \\
\hline & 2 & 85.7076 & 71.6382 & 69.1941 & 74.3049 \\
\hline & 3 & 80.6319 & 69.4405 & 68.4922 & 71.5221 \\
\hline & 4 & 75.5013 & 67.4246 & 68.6999 & 69.4460 \\
\hline & 5 & 81.4914 & 70.0634 & 69.1533 & 71.1584 \\
\hline & 6 & 84.9192 & 72.0679 & 69.4799 & 74.4547 \\
\hline & 7 & 78.2602 & 68.8239 & 67.8390 & 70.5078 \\
\hline & 8 & 84.0480 & 71.9752 & 68.6459 & 72.6823 \\
\hline & 9 & 82.6293 & 71.2381 & 69.2973 & 72.2141 \\
\hline & 10 & 77.4154 & 69.0196 & 68.5314 & 70.3796 \\
\hline
\end{tabular}

In Fig.7, the image restoration performance of our proposed APSO-RL and standard PSO-RL methods with different denoising filters comparison is given. From Fig. 7, we know that our proposed APSO-RL method has given high SDME value in all denoising filters than the PSO-FL method. But the PSO-RL based restoration acquired slight deviation from our proposed APSO-RL when the denoising filter is our proposed FMF filter. In other filtering methods our proposed APSO-RL has given high restoration performance in terms of their SDME measure. Thus, our proposed FMF with APO-PL methods has given high-quality performance in image denoising and restoration. 
Table 2. Image Restoration Results from proposed APSO-RL and PSO-RL

\begin{tabular}{|c|c|c|c|c|c|c|c|c|c|}
\hline \multirow{3}{*}{\begin{tabular}{|c|} 
Noise \\
Variance \\
$\left(\sigma^{2}\right)$ \\
\end{tabular}} & \multirow{3}{*}{ Images } & \multicolumn{4}{|c|}{ Modified PSO } & \multicolumn{4}{|c|}{ PSO } \\
\hline & & Proposed & Bilateral & Wiener & Median & Proposed & Bilateral & Wiener & Median \\
\hline & & FMF & Filter & Filter & Filter & FMF & Filter & Filter & Filter \\
\hline \multirow{10}{*}{0.02} & 1 & 45.98 & 21.76 & 34.53 & 30.31 & 44.18 & 29.65 & 36.30 & 27.71 \\
\hline & 2 & 49.05 & 37.87 & 48.00 & 35.73 & 37.18 & 35.91 & 52.74 & 29.20 \\
\hline & 3 & 36.57 & 21.24 & 42.91 & 35.72 & 35.45 & 28.47 & 38.15 & 6.81 \\
\hline & 4 & 40.91 & 31.05 & 37.73 & 32.03 & 39.37 & 24.17 & 6.65 & 34.80 \\
\hline & 5 & 33.07 & 34.97 & 42.80 & 34.63 & 37.09 & 32.03 & 50.12 & 32.48 \\
\hline & 6 & 36.36 & 33.36 & 39.09 & 22.55 & 41.20 & 16.52 & 46.60 & 46.32 \\
\hline & 7 & 38.59 & 23.79 & 27.19 & 27.84 & 39.25 & 22.73 & 40.72 & 22.49 \\
\hline & 8 & 54.56 & 23.82 & 51.45 & 26.13 & 52.49 & 20.51 & 44.92 & 36.26 \\
\hline & 9 & 65.64 & 38.26 & 45.94 & 45.48 & 58.51 & 33.75 & 34.17 & 34.09 \\
\hline & 10 & 34.10 & 29.62 & 34.30 & 23.36 & 35.48 & 23.44 & 47.22 & 24.44 \\
\hline \multirow{10}{*}{0.04} & 1 & 46.01 & 15.65 & 39.10 & 25.70 & 41.98 & 12.36 & 38.35 & 18.34 \\
\hline & 2 & 55.12 & 24.85 & 34.62 & 21.68 & 49.00 & 24.55 & 33.86 & 38.94 \\
\hline & 3 & 34.63 & 18.11 & 24.69 & 31.33 & 35.98 & 18.30 & 32.06 & 28.95 \\
\hline & 4 & 31.35 & 21.82 & 28.95 & 25.04 & 42.67 & 32.68 & 32.94 & 29.73 \\
\hline & 5 & 48.82 & 28.32 & 47.46 & 27.04 & 37.58 & 27.00 & 41.56 & 10.63 \\
\hline & 6 & 47.44 & 31.88 & 30.16 & 29.91 & 47.20 & 10.89 & 42.31 & 23.48 \\
\hline & 7 & 39.44 & 24.03 & 39.52 & 26.98 & 18.16 & 18.32 & 25.17 & 29.49 \\
\hline & 8 & 35.08 & 22.75 & 31.67 & 32.22 & 47.56 & 21.69 & 29.94 & 34.62 \\
\hline & 9 & 54.94 & 30.31 & 38.28 & 34.26 & 53.21 & 30.33 & 37.62 & 37.54 \\
\hline & 10 & 29.27 & 25.24 & 39.91 & 37.82 & 27.47 & 17.35 & 38.42 & 30.12 \\
\hline \multirow{10}{*}{0.06} & 1 & 36.19 & 21.57 & 24.35 & 19.88 & 40.27 & 18.89 & 35.04 & 20.91 \\
\hline & 2 & 54.29 & 23.12 & 26.27 & 22.93 & 54.01 & 23.68 & 17.61 & 21.79 \\
\hline & 3 & 24.76 & 26.27 & 33.74 & 22.02 & 27.95 & 15.50 & 36.12 & 20.37 \\
\hline & 4 & 28.36 & 22.25 & 39.61 & 27.40 & 33.84 & 23.74 & 32.90 & 21.05 \\
\hline & 5 & 48.53 & 15.63 & 51.35 & 28.95 & 39.02 & 18.28 & 43.64 & 22.04 \\
\hline & 6 & 41.39 & 13.44 & 21.63 & 17.31 & 39.62 & 24.29 & 29.63 & 27.04 \\
\hline & 7 & 37.93 & 27.24 & 24.21 & 11.54 & 33.12 & 22.49 & 27.45 & 17.37 \\
\hline & 8 & 47.03 & 18.50 & 23.20 & 27.95 & 47.97 & 1.57 & 29.03 & 26.41 \\
\hline & 9 & 59.68 & 26.70 & 40.89 & 29.45 & 58.50 & 7.87 & 18.26 & 30.91 \\
\hline & 10 & 32.04 & 28.45 & 34.32 & 27.06 & 27.02 & 14.42 & 34.46 & 26.09 \\
\hline \multirow{10}{*}{0.08} & 1 & 43.98 & 14.14 & 31.92 & 21.10 & 34.02 & 11.73 & 20.29 & 18.92 \\
\hline & 2 & 53.33 & 27.34 & 34.17 & 27.19 & 35.73 & 16.41 & 36.32 & 13.34 \\
\hline & 3 & 31.38 & 10.43 & 24.89 & 20.60 & 32.03 & 11.59 & 29.54 & 18.80 \\
\hline & 4 & 34.18 & 30.38 & 41.93 & 17.07 & 42.30 & 24.88 & 38.91 & 26.62 \\
\hline & 5 & 35.71 & 14.41 & 32.01 & 22.27 & 43.95 & 18.12 & 24.39 & 29.51 \\
\hline & 6 & 49.51 & 13.21 & 23.44 & 26.93 & 40.55 & 17.26 & 39.29 & 24.27 \\
\hline & 7 & 37.73 & 22.93 & 20.67 & 18.92 & 37.82 & 13.83 & 25.71 & 19.48 \\
\hline & 8 & 52.49 & 27.92 & 41.31 & 21.62 & 52.93 & 17.97 & 34.83 & 15.41 \\
\hline & 9 & 61.50 & 19.71 & 31.20 & 24.53 & 62.58 & 20.02 & 44.11 & 21.22 \\
\hline & 10 & 36.80 & 24.72 & 29.01 & 18.26 & 32.22 & 17.25 & 11.37 & 18.61 \\
\hline
\end{tabular}




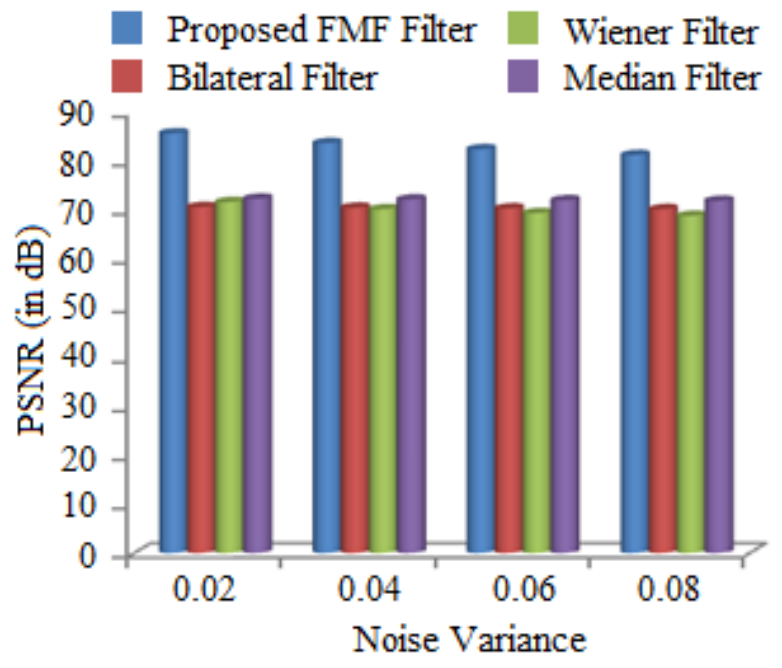

Figure.6 Average noise removal performance of our proposed FMF denoising filter and existing filtering methods

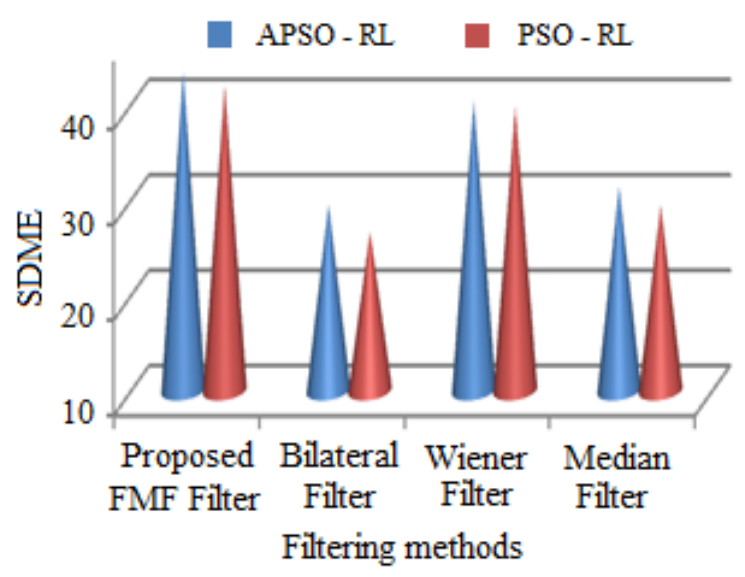

Figure.7 Average image restoration performance of our proposed APSO-RL and existing filtering methods

\section{Conclusion}

In this paper, the given (fig 4) images noise to be removed and that noise free image is to be restored by exploiting FMF and APSO-RL algorithm. By these techniques our proposed method has given high denoising and restoration performance. The implementation result shows the performance of FMF and APSO-RL methods in the denoising and restoration process in terms of their high value in PSNR and SDME than the other filtering methods. Hence, the image can be denoisied and restored more effectively by achieving higher image denoising and quality ratio rather than the conventional techniques.

Further more if we notice figure 6 and 7 the noise extraction features of proposed method is more than existing techniques and image restoration performance of our proposed APSO-RL is better than
PSO-RL.

The research work can be carried out further by employing techniques to remove noise up to high level, unless we are not successful in removing noise pixel we cannot boost noiseless pixels alone for restoration.

\section{References}

[1] S. Kim, "PDE-Based Image Restoration: A Hybrid Model and Color Image Denoising", IEEE Transactions on Image Processing, Vol. 15, No. 5, May 2006.

[2] V. N. Bushanam, G. S. V. P. Raju and Vaddimukkala Prasad, "Image Restoration and Topological Optimization", International Journal of Computer Applications, Vol. 22, No. 1, pp. 0975 - 8887, 2011.

[3] P. Chawla, R. Mittal and K. Grewal, "Hybrid Filtering Technique for Image Denoising Using Artificial Neural Network", International Journal of Engineering and Advanced Technology, Vol. 1, No. 3, pp. 2249-8958, 2012.

[4] I. Daubechies and G. Teschke, "Variational Image Restoration By Means Of Wavelets: Simultaneous Decomposition, Deblurring, and Denoising", Applied and Computational Harmonic Analysis, Vol. 19, No. 1, pp. 1-16, 2005.

[5] N. Gulati and A. Kaushik, "Remote Sensing Image Restoration Using Various Techniques: A Review", International Journal of Scientific \& Engineering Research, Vol. 3, No. 1, 2012.

[6] X. G. Lv, Y. Z. Song, S. X. Wang and J. Le, "Image restoration with a high-order total variation minimization method", Applied Mathematical Modelling, Vol. 37, No. 16, pp. 8210-8224, 2013.

[7] Y. Zhang, A. Duijster and P. Scheunders, "A Bayesian Restoration Approach for Hyperspectral Images", IEEE Transactions On Geoscience and Remote Sensing, Vol. 50, No. 9, pp. 3453-3462, 2012.

[8] T. S. Cho, C. L. Zitnick, N. Joshi, S. B. Kang, R. Szeliski and W. T. Freeman, "Image Restoration by Matching Gradient Distributions", IEEE Transactions On Pattern Analysis And Machine Intelligence, Vol. 34, No. 4, pp. 683-694, 2012.

[9] M. Jung, X. Bresson, T. F. Chan, and L. A. Vese, "Nonlocal Mumford-Shah Regularizers for Color Image Restoration", IEEE Transactions On Image Processing, Vol. 20, No. 6, pp. 1583-1598, 2011.

[10] S. K. Satpathy, S. Panda, K. K. Nagwanshi and C. Ardil, "Image Restoration in Non-Linear 
Filtering Domain using MDB approach", International Journal of Information and Communication Engineering, Vol. 6, No. 1, pp. 45-49, 2010.

[11] F. A. Jassim, "A Kalman Filter Approach for Denoising and Deblurring 3-D Microscopy Images", IEEE Transactions on Image Processing, Vol. 22, No. 12, pp. 5306-5321, 2013.

[12] J. Wang, Y. Guo, Y. Ying, Y. Liu and Q. Peng, "Fast Non-Local Algorithm For Image Denoising", In Proceedings of IEEE International Conference on Image Processing, pp. 1429 - 1432, 2006.

[13] S. G. Chang, B.Yu and M. Vetterli, "Adaptive Wavelet Thresholding for Image Denoising and Compression", IEEE Transactions On Image Processing, Vol. 9, No. 9, pp. 1532-1546, 2000.

[14] K. Dabov, A. Foi, V. Katkovnik and K. Egiazarian, "Image denoising with blockmatching and 3D filtering", In Proceedings of SPIE-IS \& T Electronic Imaging, pp. 606414606414, 2006.

[15] X. Y. Wang, H. Y. Yang, Y. Zhang and Z. K. $\mathrm{Fu}$, "Image Denoising Using SVM Classification In Nonsubsampled Contourlet Transform Domain", Information Sciences, Vol. 246, pp. 155-176, 2013.

[16] X. Y. Wang, H. Y. Yang and Z. K. Fu, "Edge Structure Preserving Image Denoising Using OAGSM/NC Statistical Model”, Digital Signal Processing, Vol. 23, No. 1, pp. 200-212, 2013.

[17] B. Li and D. Que, "Medical Images Denoising Based on Total Variation Algorithm", In Proceedings of International Conference on Environment Science and Biotechnology, Vol. 8, pp. 227-234, 2011.

[18] S. Khan, A. Jain and A. Khare, "Denoising of Images Based on Different Wavelet Thresholding by Using Various Shrinkage Methods using Basic Noise Conditions", International Journal of Engineering Research \& Technology, Vol. 2, No. 1, 2013.

[19] H. Zhang, J. Yang, Y. Zhang and T. S. Huang, "Image and Video Restorations via Nonlocal Kernel Regression", IEEE Transactions on Cybernetics, Vol. 43, No. 3, pp. 1035-1046, 2013.

[20] M. Elad and M. Aharon, "Image Denoising Via Sparse and Redundant Representations Over Learned Dictionaries", IEEE Transactions On Image Processing, Vol. 15, No. 12, pp. 37363745, 2006.

[21] M. K. Ng, X. Yuan and W. Zhang, "Coupled Variational Image Decomposition and Restoration Model for Blurred Cartoon-Plus-
Texture Images With Missing Pixels", IEEE Transactions On Image Processing, Vol. 22, No. 6, pp. 2233-2246, 2013.

[22] W. Dong, L. Zhang, G. Shi and X. Li, "Nonlocally Centralized Sparse Representation for Image Restoration", IEEE Transactions On Image Processing, Vol. 22, No. 4, pp. 1620-1630, 2013.

[23] A. Rajwade, A. Rangarajan and A. Banerjee, "Image Denoising Using the Higher Order Singular Value Decomposition", IEEE Transactions On Pattern Analysis And Machine Intelligence, Vol. 35, No. 4, pp. 849-862, 2013.

[24] A. Fathi and A. R. N. Nilchi, "Efficient Image Denoising Method Based on a New Adaptive Wavelet Packet Thresholding Function", IEEE Transactions on Image Processing, Vol. 21, No. 9, pp. 3981-3990, 2012.

[25] T. S. Cho, C. L. Zitnick, N. Joshi, S. B. Kang, R. Szeliski, and W. T. Freeman, "Image Restoration by Matching Gradient Distributions", IEEE Transactions On Pattern Analysis And Machine Intelligence, Vol. 34, No. 4, pp. 683-694, 2012.

[26]P. Zhang and F. Li, "A New Adaptive Weighted Mean Filter for Removing Salt-and-Pepper Noise", IEEE Signal Processing Letters, Vol. 21, No. 10, October 2014.

[27]V. B. S. Prasath, D. Vorotnikov, R. Pelapur, S. Jose, G. Seetharaman and K. Palaniappan, "Multiscale Tikhonov-Total Variation Image Restoration Using Spatially Varying Edge Coherence Exponent", IEEE Transactions On Image Processing, Vol. 24, No. 12, December 2015.

[28] H. Zhang, W. He, L. Zhang, H. Shen and Q. Yuan,", IEEE Transactions On Geoscience And Remote Sensing, Vol. 52, No. 8, 2014.

[29]A. Roy, J. Singha, S. S. Devi and R. H. Laskar, "Impulse noise removal using SVM classification based fuzzy filter from Gray scale images", Elsevier inc, Vol. 128, pp. 262-273, 2016.

[30] R. Dash, B. Majhi, "Motion blur parameters estimation for image restoration", Elsevier inc, Vol. 125, No. 5, pp. 1634-1640, 2014. 